\title{
Polarized Lanthanum Target for the T-violation Search in Slow Neutron Transmission
}

Kohei Ishizaki*, Hirohiko M. Shimizu, Masaaki Kitaguchi, Taku Matsushita

Nagoya University, Nagoya, Aichi, 464-8602, Japan

E-mail: ishizakiephi.phys.nagova-u.ac.ip,

\section{Masataka linuma}

Hiroshima University, Higashi-hiroshima, Hiroshima, 739-8530, Japan

Hideki Kohri, Hiromoto Yoshikawa, Masaru Yosoi, Tatsushi Shima

Research Center for Nuclear Physics, Osaka University, Ibaraki, Osaka, 567-0047, Japan

\section{Takahiro Iwata, Yoshiyuki Miyachi}

Yamagata University, Yamagata, Yamagata, 990-8560, Japan

\section{Shigeru Ishimoto}

KEK, Tsukuba, Ibaraki, 305-0801, Japan

\section{Masaki Fujita, Yoichi Ikeda}

Institute for Materials Research, Tohoku University, Sendai, Miyagi, 980-8577, Japan

\begin{abstract}
The $\mathrm{Nd}^{3+}$ doped $\mathrm{LaAlO}_{3}$ crystal is a good candidate of a polarized ${ }^{139} \mathrm{La}$ target for the search of the P-odd and T-odd interaction in the low-energy neutron absorption of ${ }^{139} \mathrm{La}$. As the first step for realizing the polarized ${ }^{139}$ La target, we grow the undoped $\mathrm{LaAlO}_{3}$ crystal and the $\mathrm{La}_{1-x} \mathrm{Nd}_{x} \mathrm{AlO}_{3}$ $\left(x=5 \times 10^{-4}\right)$ crystal by floating-zone method with four halogen lamps. The ESR measurements of $\mathrm{Nd}^{3+}$ in the latter crystal indicated that the linewidth was about 6 gauss, whose value is the same as the crystal in which $50 \%{ }^{139}$ La polarization was achieved in the previous DNP experiment. As the result, the crystals grown by this method can be applicable to the target study for the T-violation search.
\end{abstract}

The 18th International Workshop on Polarized Sources, Targets, and Polarimetry, PSTP2019 23-27 September, 2019

Knoxville, Tennessee

\footnotetext{
* Speaker.
} 


\section{Introduction}

The ratio of amount of matter to anti-matter in the universe can be predicted from the CabibboKobayashi-Maskawa(CKM) model, but there is still a large discrepancy from the observational cosmology [四]. One possible explanation is the existence of undiscovered CP violation in the fundamental interactions [వ].

In the ${ }^{139} \mathrm{La}$, the P-violating asymmetry in the polarized low-energy neutron absorption is about six orders of magnitude larger than that in the nucleon-nucleon scattering [B], 田]. A theory also predicts the large enhancement of the P-odd and T-odd asymmetry in the reaction [[]]. According to the CPT theorem, the measurement of T-violating effects is applicable to search the unknown $\mathrm{CP}$ violating interaction. In the above system, an effect of the P-odd and T-odd appears as a contribution of $\vec{\sigma} \cdot(\vec{I} \times \vec{k})$ in the forward scattering amplitude, where $\vec{\sigma}, \vec{I}$, and $\vec{k}$ are a neutron spin, a nuclear spin of the target, and a neutron momentum, respectively. In experimental configuration sensitive to the P-odd and T-odd asymmetry, a spin rotation of the incident neutron is unavoidable because of a pseudomagnetic field induced by the polarized nuclei [ $]$ ]. The field strength in the $\mathrm{LaAlO}_{3}$ crystal is estimated to be roughly less than $0.2 \mathrm{~T}$ [ [ $\mathrm{C}$, 目]. If we apply the external magnetic field as low as the psudomagnetic field, it is expected to cancel out the effect of the psudomagnetic rotation.

The NOPTREX ( Neutron Optics for Parity and Time Reversal EXperiment ) collaboration is planning to make the precise measurement of the T-violating effects in the absorption of the low energy neutrons. Our previous investigations with a detection of emitted gamma rays have revealed that the ${ }^{139} \mathrm{La}$ nucleus is one of candidates for the target nuclei [ $[$ ]. Moreover, about $50 \%{ }^{139} \mathrm{La}$ polarization in the single crystal of $\mathrm{LaAlO}_{3}$ doped with $\mathrm{Nd}$ ions has been achieved by the conventional DNP in PSI [U0]. These are reasons why we have chosen the ${ }^{139} \mathrm{La}$ for the first experimental exploration.

The $\mathrm{LaAlO}_{3}$ crystals have preferable characteristics as the target material. First, other kinds of nuclei in the crystal, ${ }^{16} \mathrm{O}$ and ${ }^{27} \mathrm{Al}$ have very low absorption compared to that of the ${ }^{139} \mathrm{La}$ nuclei. Secondary, the symmetrical axises of the electrical field gradient around each nucleus are aligned to the $C_{3}$ axis. This feature allows us to diagonalize the quadruple coupling along the $C_{3}$ axis. Therefore, if the magnetic field is parallel to the $C_{3}$ axis, the reduction of the magnetic field is in principle possible with keeping the high polarization.

In general, the DNP needs paramagnetic centers as a seed of highly electron polarization and a narrow linewidth of ESR spectrum is preferable for an efficient polarization transfer. Since the $\mathrm{Nd}^{3+}: \mathrm{LaAlO}_{3}$ crystal has one magnetically equivalent site for La nuclei and $\mathrm{Nd}$ ions, the ESR linewidth is very narrow. This feature has a great advantage in performing the DNP [प]].

Toward the realization of the polarized ${ }^{139} \mathrm{La}$ target for a practical use, we have some issues on the $\mathrm{Nd}^{3+}: \mathrm{LaAlO}_{3}$ crystals. Inhomogeneity of local magnetic field at the La site must be suppressed so that the ESR line width is sufficiently narrow relative to the nuclear Zeeman splitting and well-resolved solid effect can be expected. The twin domain, which easily appears due to the structural phase transition from cubic to trigonal at $813 \mathrm{~K}$ for $\mathrm{LaAlO}_{3}$ [ए2], should be removed as much as possible. Additionally, the density of the paramagnetic center should be optimized by comparing the efficiency of the polarization buildup in the DNP and the spin-lattice relaxation rate. These requirements should be satisfied in sufficiently large crystal for the T-violation experiment, 
in which the preferred crystal size is $4 \mathrm{~cm} \times 4 \mathrm{~cm} \times 5 \mathrm{~cm}$.

As the beginning stage on the preparation of the crystals, we have studied the crystal growth for a small size of the $\mathrm{Nd}^{3+}: \mathrm{LaAlO}_{3}$ crystals and evaluated the grown crystals through the ESR spectrum.

\section{Crystal growth of $\mathrm{LaAlO}_{3}$}
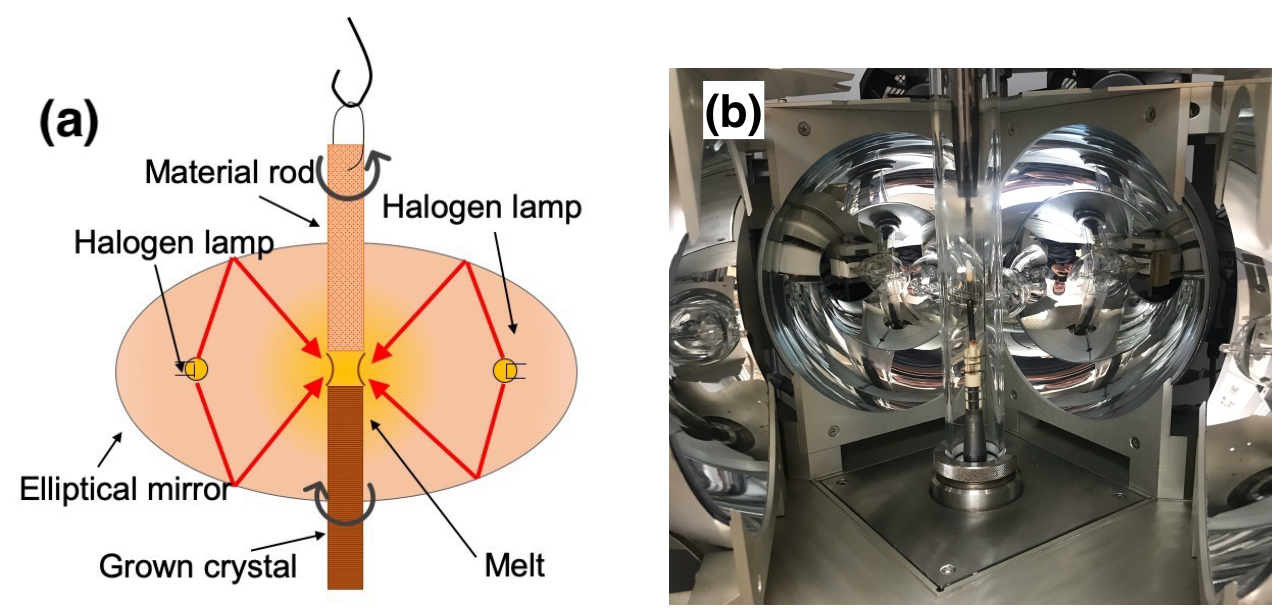

Figure 1: (a) : Illustration of floating-zone method. The details are written in the main text. (b) : Floatingzone furnace with four elliptical mirrors, which was used in the crystal growth.

We tried to make the crystal growth of $\mathrm{LaAlO}_{3}$ by floating zone method with halogen-lamp (Figure $\mathbb{W}$ ). In the method, the material of hanged rod is molten by intensively irradiating the infrared light with elliptical mirrors. Molten material is crystallized when the melting material is cooled down by traveling it upward from the zone in which the infrared light is collected with the mirrors. This method has a merit that a dopant concentration is easily controlled. In the Czochralski method, for example, a segregation easily appears, although it is useful for growing large crystals. For the purpose of growing small crystals, the floating zone method is widely used for researchers, especially, in the field of solid state physics.

We used the floating-zone furnace made by Crystal Systems Corporation with four halogen lamps. Since the melting point of $\mathrm{LaAlO}_{3}$ is about $2100{ }^{\circ} \mathrm{C}$, almost all growth of $\mathrm{LaAlO}_{3}$ by floating-zone method was conducted with high-power xenon lamps. Therefore, the furnace was used with the maximum output power $(4 \mathrm{~kW})$ to melt the $\mathrm{LaAlO}_{3}$ rod. First, we synthesized the powder of $\mathrm{LaAlO}_{3}$ by calcining raw materials, $4 \mathrm{~N} \mathrm{La}(\mathrm{OH})_{3}$ and $\mathrm{Al}_{2} \mathrm{O}_{3}$, at $1400{ }^{\circ} \mathrm{C}$ for 8 hours. After that, the material rod was prepared by the hydrostatic molding of the calcined $\mathrm{LaAlO}_{3}$ powder and the sintering at $1400{ }^{\circ} \mathrm{C}$ for 8 hours more. Additionally, the rod was sintered using the floatingzone furnace with a traveling speed of $5 \mathrm{~mm} / \mathrm{h}$ before the growing process.

We grow the crystals with the speed of $10 \mathrm{~mm} / \mathrm{h}$ in the atmosphere. As the results, we obtained the following $\mathrm{LaAlO}_{3}$ crystals, undoped(\#1), $0.05 \% \mathrm{Nd}$ doped (\#2), and undoped and grown along

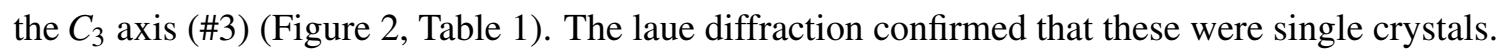

The undoped crystal (\#1) was cut into two pieces, and one of them was annealed at $1200{ }^{\circ} \mathrm{C}$ for 60 hours in the atmosphere. This process changed the color of the crystal from dark brown to 
transparent brown (Figure [B]). It may be caused by changing the valence of impurity ions in the crystal.

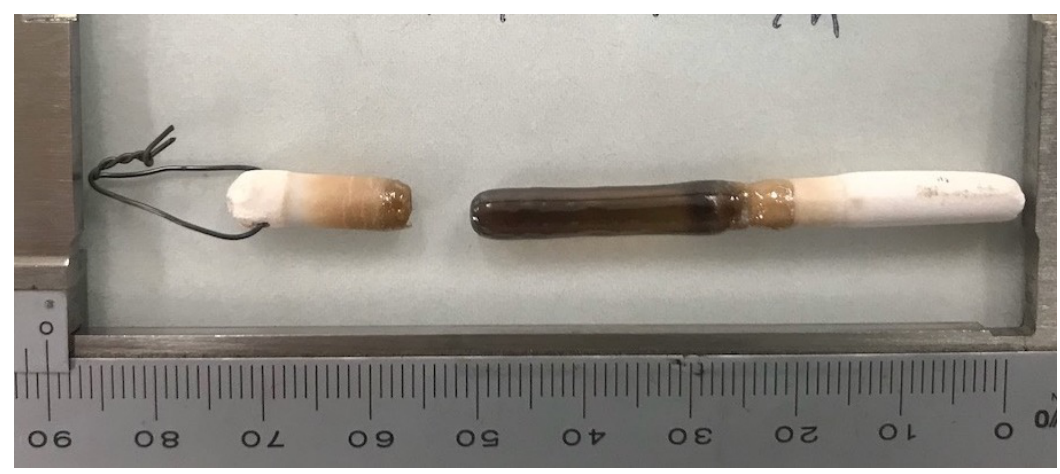

Figure 2: Undoped $\mathrm{LaAlO}_{3}$ crystal (5 $\mathrm{mm}$ in diameter and $20 \mathrm{~mm}$ in length). The growth was implemented under the condition : the growth direction was $<111>_{\text {cubic }}$ and the growth speed was $10 \mathrm{~mm} / \mathrm{h}$.

\begin{tabular}{|c|c|c|c|}
\hline $\begin{array}{c}\text { sample } \\
\text { number }\end{array}$ & diameter & dopant & remarks \\
\hline \hline$\# 1 \mathrm{a}$ & $5 \mathrm{~mm}$ & none & No annealing \\
$\# 1 \mathrm{~b}$ & $5 \mathrm{~mm}$ & none & annealing for 60 hours at $1200{ }^{\circ} \mathrm{C}$ in the atmosphere \\
$\# 2$ & $5 \mathrm{~mm}$ & $\mathrm{Nd} 0.05 \%$ & No annealing \\
$\# 3$ & $5 \mathrm{~mm}$ & none & growing along the $C_{3}$ axis. \\
\hline
\end{tabular}

Table 1: Summary of the crystals grown by the floating zone method

\section{ESR measurement of $\mathrm{Nd}$ doped $\mathrm{LaAlO}_{3}$}

The ESR spectra of the \#2 crystal, $\mathrm{La}_{1-x} \mathrm{Nd}_{x} \mathrm{AlO}_{3}\left(x=5 \times 10^{-4}\right)$, were measured by the CWESR apparatus to check the possibility of the DNP. The temperature of the crystal was kept to 15 $\mathrm{K}$ and the microwave frequency was $9.493 \mathrm{GHz}$. We also measured the angular dependence of the ESR spectrum.

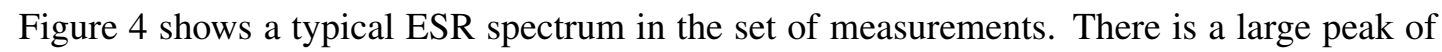
$\mathrm{Nd}^{3+}$ around $0.3165 \mathrm{~T}$. The angular dependence of its resonance frequency leads to the g-factors of $g_{\|}=2.14$ and $g_{\perp}=2.66$ and its linewidth is about 6 gauss. These values are consistent with the ones in the reference [U]]. Particularly, the ESR width and the Nd concentration are almost the same as that of the crystal used in the past DNP, which shows the achievement of about $50 \%{ }^{139} \mathrm{La}$ polarization [ए]]. Therefore, the grown crystal (\#2) is expected to indicate the large enhancement by the DNP. Incidentally, the linewidth is explained by the superhyperfine between the $\mathrm{Nd}^{3+}$ ion and the eight neighboring ${ }^{27} \mathrm{Al}$ nuclei [ए]].

In contrast, some small peaks can be seen in the low field region. It can be explained that these peaks are originated from the alternative domains, because the resonance frequency is consistent with the estimation from the obtained g-factors and the orientation of $C_{3}$ axis of the other domains. 


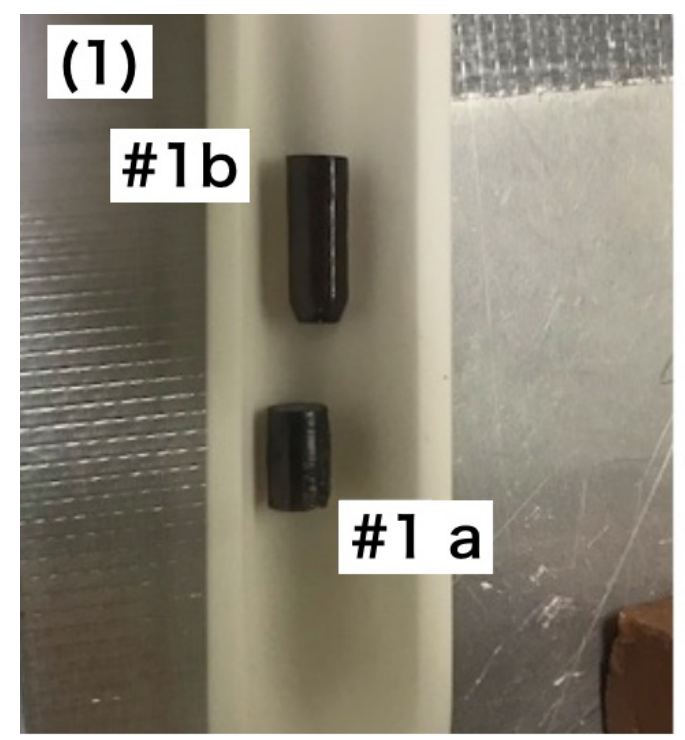

\section{(2)}

\# 1 b

\#1 a

Figure 3: Annealing of the undoped $\mathrm{LaAlO}_{3}$ crystal. (1) : Before annealing, (2) : The crystal \#1 b had been annealed for 60 hours at $1200{ }^{\circ} \mathrm{C}$ in the atmosphere.

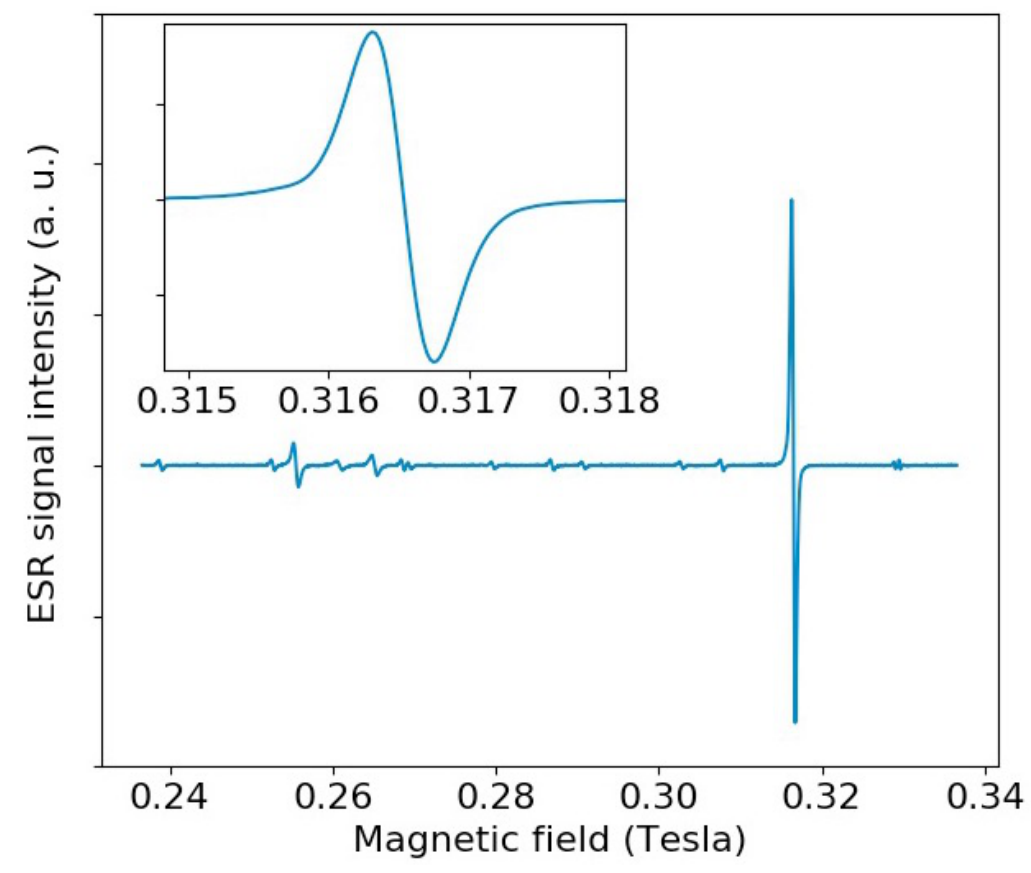

Figure 4: One of the ESR spectrums of the sample \# 2 in the Table $\mathbb{U}$. In this measurement, the $C_{3}$ axis was aligned to the magnetic field. 


\section{Summary and prospects}

The NOPTREX collaboration is planning to make the precise measurement of the T-violation in the polarized neutron absorption of the polarized ${ }^{139} \mathrm{La}$ nucleus. We are proceeding to the development of the ${ }^{139} \mathrm{La}$ polarized target. One of significant issues is to prepare the target material for the DNP test, which is the single crystal of $\mathrm{LaAlO}_{3}$ doped with $\mathrm{Nd}^{3+}$ ions. The small crystals were grown by the floating zone method under the various conditions and the ESR measurements were performed with one of the grown crystals, $\mathrm{La}_{1-x} \mathrm{Nd}_{x} \mathrm{AlO}_{3}\left(x=5 \times 10^{-4}\right)$. The results have shown that the ESR linewidth as well as the Nd concentration is almost same as that of the crystal examined in the past DNP. Therefore, the crystal grown by the floating zone method is promising for obtaining the large enhancement by the DNP, although it has twin domains. The study for reducing the twinning is progressing with the grown crystals.

The preparation for the DNP tests is in progress at Research Center for Nuclear Physics, Osaka University. Achieving the sufficiently high polarization to satisfy the practical use, we are going to study the DNP and investigate the dependence of the spin-lattice relaxation on the concentration of $\mathrm{Nd}^{3+}$ with the grown crystals. The T-violation experiment also requires the large crystals, whose growth may be feasible by using the other growth methods with the crucible [143, [4]]. We will grow the large crystal in the Institute for Materials Research, Tohoku University.

\section{Acknowledgments}

We thank to Dr. H. Mino for the ESR measurement, and Dr. H. Taniguchi for the annealing process. The studies on the target material were supported by Tohoku University IMR cooperative program(proposal No. 18G0034, 19K0081, and 19G0037) and JSPS KAKENHI Grant Number JP17H02889. The study of the DNP technique was supported by the RCNP project "Development of polarized target for new physics search via T-violation" and KEK Neutron Science Division S-type program number 2018S12.

\section{References}

[1] M. Peskin, The matter with antimatter, Nature $419(2002) 25$.

[2] A. D. Sakharov, Violation of CP invariance, $C$ asymmetry, and baryon asymmetry of the universe, Soviet Physics Uspekhi 34 (1991) 392.

[3] V. Alfimenkov, S. Borzakov, V. Van Thuan, Y. Mareev, L. Pikelner, A. Khrykin et al., Parity nonconservation in neutron resonances, Nuclear Physics A 398 (1983) 93.

[4] G. Mitchell, J. Bowman, S. Penttilä and E. Sharapov, Parity violation in compound nuclei: experimental methods and recent results, Physics Reports 354 (2001) 157.

[5] V. Gudkov, On CP violation in nuclear reactions, Physics Reports 212 (1992) 77.

[6] A. Abragam and M. Goldman, Nuclear magnetism: order and disorder, International series of monographs on physics. Clarendon Press, 1982.

[7] Y. Takahashi, H. M. Shimizu, T. Maekawa and T. Yabuzaki, A new design of a polarized target for the test of time reversal invariance in a neutron transmission experiment, Physics Letters B 326 (1994) 27. 
[8] V. Gudkov and H. M. Shimizu, Pseudomagnetic effects for resonance neutrons, Physical Review C 95 (2017)045501.

[9] T. Okudaira, S. Takada, K. Hirota, A. Kimura, M. Kitaguchi, J. Koga et al., Angular distribution of $\gamma$ rays from neutron-induced compound states of ${ }^{140}$ La, Physical Review C 97 (2018) 034622.

[10] P. Hautle and M. Inuma, Dynamic nuclear polarization in crystals of $\mathrm{Nd}^{3+}: \mathrm{LaAlO}_{3}$, a polarized ${ }^{139}$ La target for a test of time-reversal invariance, Nuclear Instruments and Methods in Physical Research A 440 (2000) 638.

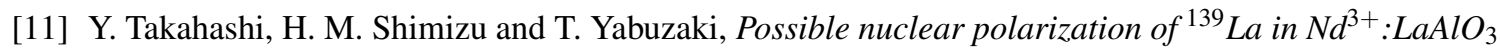
for the test of time reversal invariance, Nuclear Instruments and Methods in Physics Research A 336 (1993) 583 .

[12] S. A. Hayward, F. D. Morrison, S. A. T. Redfern, E. K. H. Salje, J. F. Scott, K. S. Knight et al., Transformation processes in $\mathrm{LaAlO}_{3}$ : Neutron diffraction, dielectric, thermal, optical, and Raman studies, Physical Review B 72(2005) 054110.

[13] G. Berkstresser, A. Valentino and C. Brandle, Growth of single crystals of lanthanum aluminate, Dournal of Crystal Growth 109 (1991) 467.

[14] R. E. Fahey, A. J. Strauss and A. C. Anderson, Vertical gradient-freeze growth of aluminate crystals, Dournal of Crystal Growth 128 (1993) 672. 